\title{
Kalman Filter Algorithm for Adaptive Digital Predistortion
}

\author{
Xiaowei Kong, Jinzheng Li, Wei Xia and Zishu He \\ School of Electronic Engineering \\ University of Electronic Science and Technology of China \\ Chengdu, China \\ Email: xiaoweikong@tom.com
}

\begin{abstract}
This paper introduces a recursive algorithm of Kalman filter for digital predistorter parameters extraction based on memory polynomials predistorter model. The predistorter model is firstly formulated as linear regression expression. Then we derive the state-space equation of the model and attain the steps of the algorithm. Finally, the accuracy and stability of the algorithm is proved by simulation.
\end{abstract}

Key words - digital predistorter, memory-polynomials model, Kalman filter, state-space equation, LMS

\section{INTRODUCTION}

In modern radar and wireless communication system, various non-constant envelope modulation techniques are presented to efficiently use frequency spectrum resource. The modulation signal generally has the attribute of higher peak to average power ratio (PAPR) in time domain and wider band width in frequency domain, which may have the power amplifier (PA) driven into nonlinearity region. The PA will shows the memory distortion (also is called linear distortion) and nonlinear distortion, the transmitted signal therefore will suffer from a serious in-band and out-band distortion [1-4]. So the PA linearization is unavoidable and essential task to compensate the PA distortion and meet spectrum emission requirements.

On the signal transmitter, the digital predistorter (DPD) produces the inverse nonlinearity of power amplifier (PA) to cancel the PA distortion. Very rapid convergence of parameter extraction algorithm for the predistorter model is a requirement of the predistorter design. The Kalman filter[5], which is one of the fast adaptive algorithm based on minimum mean square error criterion, is superior to the conventional LMS and RLS algorithm in algorithm stability and convergence rate.

Recently, many predistorter models, such as memory polynomials model, Hammerstein model, Wiener model, NARMA model and so on, are proposed in literature[3, 6, 7]. The paper takes memory-polynomials model as an example to mainly focus on the application of Kalman filter in the predistorter estimation. The paper is organized as follows. Section II introduces the linear regression expression of DPD model. The state-space equation of DPD model is derived from the linear regression expression in section III. The section also concludes the steps of the algorithm. Through the section, many DPD models which have the linear regression expression, not limited for the memory-polynomials model, can apply to the algorithm. And then, the section IV validates the convergence performance of Kalman filter for different input signal and the compensation ability of the DPD. Finally, in section $\mathrm{V}$, conclusions are given.

\section{LINEAR REGRESSION EXPRESSION OF DPD MODEL}

The complex-valued polynomials model, especially the memory polynomials model, is one of the most common functions for amplifier and predistorter modeling. The memory-polynomials model proposed by $\operatorname{Kim}[8]$ and Ding[9], can be expressed as

$$
y(n)=\sum_{q=0}^{Q} \sum_{k=1}^{K} a_{k q} x(n-q)|x(n-q)|^{k-1}
$$

where $x(n), y(n)$ are the input and output of model, $a_{k q}$ is the weight coefficient, $K$ is the order of the polynomials and $Q$ is the maximum memory depth. Then the (1) can be expressed in a vector notion as

$$
y(n)=\boldsymbol{\mu}^{T}(n) \boldsymbol{\omega}
$$

where $\boldsymbol{\omega}$ is a $\mathrm{K}(\mathrm{Q}+1) \times 1$ complex-valued weight vector as

$$
\boldsymbol{\omega}=\left[\begin{array}{lllllll}
a_{10} & \cdots & a_{K 0} & \cdots & a_{1 Q} & \cdots & a_{K Q}
\end{array}\right]^{T}
$$

and $\boldsymbol{\mu}(n)$ is basis vector,

$$
\begin{aligned}
& \boldsymbol{\mu}(n)=\left[\begin{array}{lllllll}
x^{10}(n) & \cdots & x^{K 0}(n) & \cdots & x^{1 Q}(n) & \cdots & x^{K Q}(n)
\end{array}\right]^{T} \\
& x^{p j}(k)=x(k-j)|x(k-j)|^{p-1}
\end{aligned}
$$

the superscript $T$ denotes vector transpose.

In the section, we are only simply reintroducing the existing model and the later section mainly focuses on the algorithm of parameters extraction and update.

\section{KALMAN FILTER ALGORITHM}

The weight vector $\boldsymbol{\omega}$ is a stable value when the input signal and the expected output signal are stationary signal or 
similar stationary signal. In other words, the error plane has unquie extreme point. When the algorithm is convergence, we can have

$$
\boldsymbol{\omega}_{k}=\boldsymbol{\omega}_{k-1}
$$

where $\boldsymbol{\omega}_{k}$ represents the weight vector at time $\mathrm{k}$. At the moment, the error between actual value and estimate value is

$$
e(k)=y(k)-\hat{y}(k)=y(k)-\boldsymbol{\mu}^{T}(k) \boldsymbol{\omega}_{k}
$$

So the $y(k)$ is

$$
y(k)=e(k)+\boldsymbol{\mu}^{T}(k) \boldsymbol{\omega}_{k}
$$

We can assume the (5) is process equation and (7) is measurement equation, then the state-space equation is

$$
\left\{\begin{aligned}
\boldsymbol{\omega}_{k} & =\boldsymbol{\omega}_{k-1} \\
y(k) & =e(k)+\boldsymbol{\mu}^{T}(k) \boldsymbol{\omega}_{k}
\end{aligned}\right.
$$

where $\boldsymbol{\omega}_{k}$ is state vector, the definition as (3), $y(k)$ is measurement value.

According to the adaptive filter theory[5, 10], we can attain the steps as follows.

Known parameters:

state transition matrix $\mathbf{F}(k, \quad k-1)=\mathbf{I} \in \mathbb{C}^{K(Q+1) \times K(Q+1)}$,

state noise vector $\quad \boldsymbol{v}_{1}(n)=\mathbf{0}$,

measurement matrix $\mathbf{C}(k)=\boldsymbol{\mu}^{T}(k) \in \mathbb{C}^{1 \times K(Q+1)}$,

measurement noise $v_{2}(k)=e(k)$

Initial : $\quad \boldsymbol{\omega}_{0}=\mathbf{0} \in \mathbb{C}^{K(Q+1) \times 1}, \quad \mathbf{P}(0)=\mathbf{I} \in \mathbb{C}^{K(Q+1) \times K(Q+1)}$

, $Q_{2}(k)=0.005$ (Based on performance metric)

Step 1: $k=1,2, \cdots, \quad N, k$ is the iteration times

$\alpha(k)=y(k)-\mathbf{C}(k) \omega_{k-1}$

$A(k)=\mathbf{C}(k) \mathbf{P}(k-1) \mathbf{C}^{H}(k)+Q_{2}(k)$

$\mathbf{K}(k)=\mathbf{P}(k-1) \mathbf{C}^{H}(k) A^{-1}(k) \in \mathbb{C}^{K(Q+1) \times 1}$

$\hat{\boldsymbol{\omega}}_{k}=\hat{\boldsymbol{\omega}}_{k-1}+\mathbf{K}(k) \alpha(k)$

$\mathbf{P}(k)=[I-\mathbf{K}(k) \mathbf{C}(k)] \mathbf{P}(k-1) \in \mathbb{C}^{K(Q+1) \times K(Q+1)}$

Step 2: $k=k+1$, goto step 1 .

\section{Simulation RESUlTS}

In this section, the simulation, which bases on the signal from the factual PA and adopts indirect learning architecture, illustrates the performance of proposed algorithm. The hardware experiment setup is shown in Figure.6. The experimental test bench mainly has one Altera EP2S60 FPGA chip, R\&S FSP and PC with MATLAB software. The device-under-test (DUT) considered in the study is a $1 \mathrm{~W}$ peak power amplifier, operated around $505 \mathrm{MHz}$. The amplifier is driven by eight tone signal and CDMA signal with $5 \mathrm{MHz}$ bandwidth. The attenuator (Att) and coupler are used for energy and flow control of signal. The memory-polynomials predistorter, loop-delay estimation unit, digital up converter
(DUC) cell and digital down converter (DDC) cell can be implemented on a single FPGA chip, which the weight coefficient of predistorter is calculated by the Kalman filter algorithm on PC. And the spectrum analyzer is adopted as digtal acquisition module which transfer digital signal to PC by the bus.

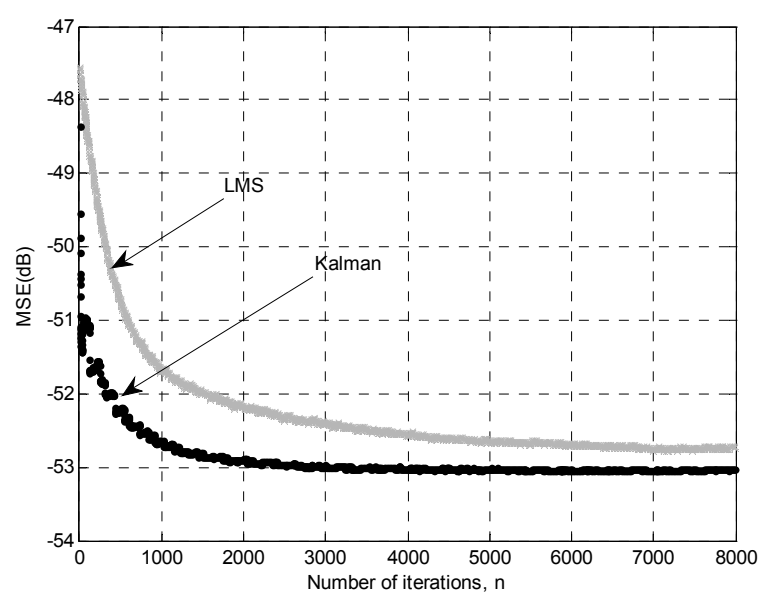

Figure.1 The learning curves of LMS and Kalman: the input is eight tone signal, the update step-size of LMS is 0.05, 400 independent trials.

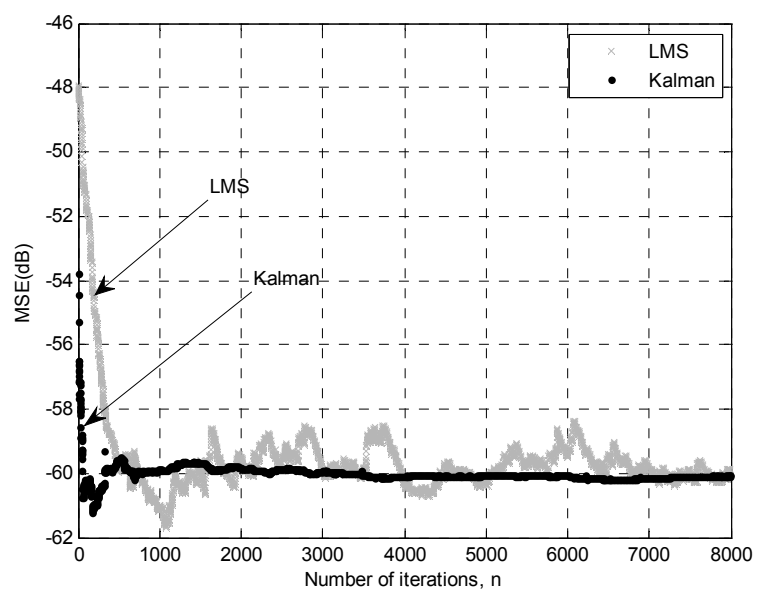

Figure.2 The learning curves of LMS and Kalman: the input is CDMA signal, the update step-size of LMS is 0.05, 400 independent trials.

Through analysis of the proposed algorithm steps in detail, we will find that the algorithm is similar to variable step size LMS. So the convergence rate will faster than conventional LMS. And Kalman filter algorithm is robust, because it not only applies to stationary random signal but also applies to non-stationary signals. Figure. 1 and Figure. 2 show the learning curves of Kalman filter algorithm and conventional LMS algorithm for different input signal. As shown in the figures, the convergence performance, especially in convergence rate, of proposed algorithm is better than conventional LMS. Figure. 3 and Figure.4 display the $\mathrm{AM} / \mathrm{AM}$ and $\mathrm{AM} / \mathrm{PM}$ curve changes between the PA without DPD and PA with DPD. The gain and phase curve become finer and straighter, because the DPD compensates the 
memory and nonlinearity distortion of amplifier. And the compensation gain is lower than the average gain before predistortion, but the linearization system can work at saturation zone of amplifier. The power spectrum comparison for the CDMA signal among input signal, distortion signal and compensation signal is shown in the Figure.5. Form the figure, we can see the improvement of adjacent channel power ratio (ACPR)[6] is greater than $20 \mathrm{~dB}$ when the predistorter is worked.

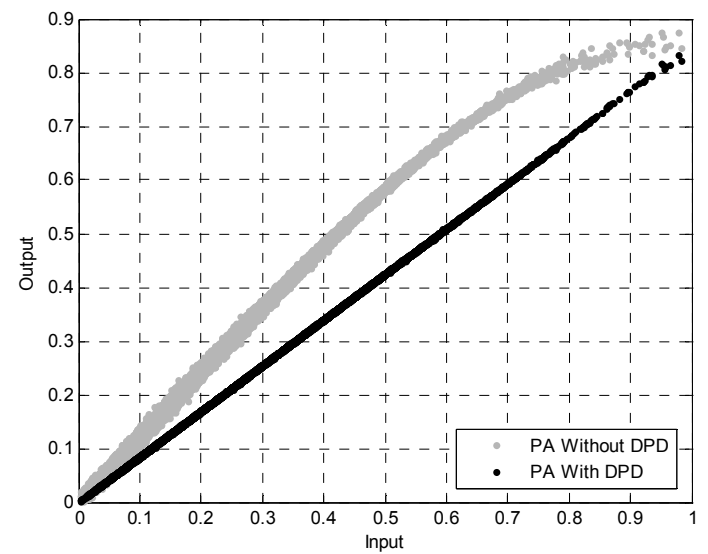

Figure.3 Amplification curve of PA (AM/AM).

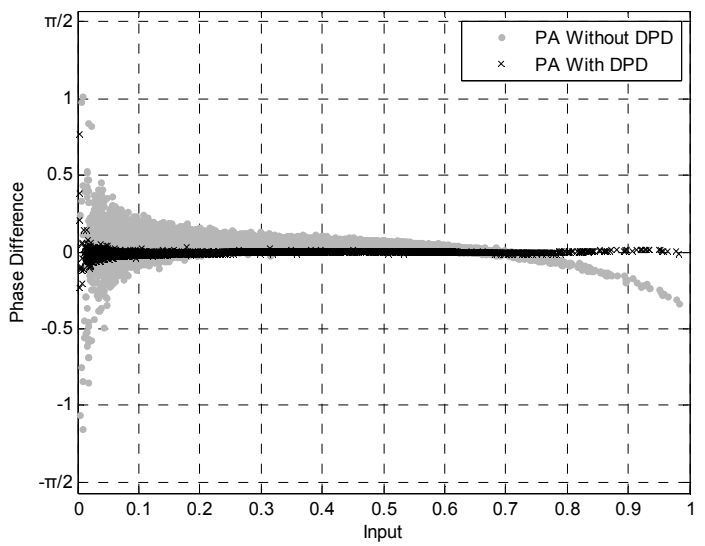

Figure.4 Phase difference of PA (AM/PM)

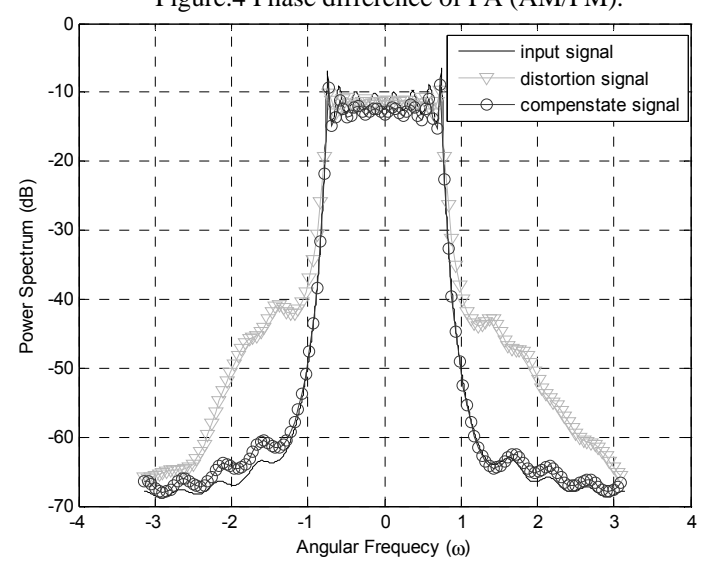

Figure.5 Power spectrum comparison.

\section{CONCLUSION}

The paper introduces an algorithm for the extraction and update of the predistorter parameters. Through the algorithm, we can get the predistorter parameters in the training phase and update the parameters based on a certain criterion, such as periodic time interval or specified performance requirement (e.g., spectrum emission mask), in the working phase. As mentioned above, the algorithm has more advantages than conventional LMS in convergence rate and stability. But the computational complexity of the algorithm is proportional to the square of $\mathrm{N}$, because the Kalman gain $\mathbf{K}(n)$ is a N-by-N matrix. For the further reducing the computational complexity and ensuring the numerical stability, we have two methods can be adopted. The first method, we can apply the the extension of Kalman filter algorithm, such as Fast Kalman filter or Square-root Kalman filter algorithm, in algorithm level. And retaining the odd order and removing even order of polynomials in model design level is the another method.

\section{ACKNOWLEDGEMENT}

This work is supported by the Fundamental Research Funds for the Central Universities of China (No. ZYGX2010J020), the Guangdong and Hong Kong major breakthrough in key fields project (No.200920523300005) and the Sichuan Key Technology Support Program (No. 2010GZ0149)

\section{REFERENCES}

[1] J. C. Pedro and S. A. Maas, "A comparative overview of microwave and wireless power-amplifier behavioral modeling approaches," Microwave Theory and Techniques, IEEE Transactions on, vol. 53, pp. 1150-1163, 2005.

[2] K. Hyunchul and J. S. Kenney, "Behavioral modeling of nonlinear RF power amplifiers considering memory effects," Microwave Theory and Techniques, IEEE Transactions on, vol. 51, pp. 2495-2504, 2003.

[3] M. Isaksson, D. Wisell, and D. Ronnow, "A comparative analysis of behavioral models for RF power amplifiers," Microwave Theory and Techniques, IEEE Transactions on, vol. 54, pp. 348-359, 2006.

[4] L. Yong-Sub, L. Mun-Woo, and J. Yoon-Ha, "A Wideband Analog Predistortion Power Amplifier With Multi-Branch Nonlinear Path for Memory-Effect Compensation," Microwave and Wireless Components Letters, IEEE, vol. 19, pp. 476-478, 2009.

[5] S. Haykin, Adaptive Filter Theory, Fourth ed. Beijing: Publishing House of Electronics Industry, 2002.

[6] P. L. Gilabert, A. Cesari, G. Montoro, E. Bertran, and J. M. Dilhac, "Multi-Lookup Table FPGA Implementation of an Adaptive Digital Predistorter for Linearizing RF Power Amplifiers With Memory Effects," Microwave Theory and Techniques, IEEE Transactions on, vol. 56, pp. 372-384, 2008.

[7] M. Djamai, S. Bachir, and C. Duvanaud, "Kalman filtering algorithm for on-line memory polynomial predistortion," in Microwave Conference, 2008. EuMC 2008. 38th European, 2008, pp. 575-578.

[8] J. Kim and K. Konstantinou, "Digital predistortion of wideband signals based on power amplifier model with memory," Electronics Letters, vol. 37, pp. 1417-1418, 2001.

[9] L. Ding, G. T. Zhou, D. R. Morgan, Z. Ma, J. S. Kenney, J. Kim, and C. R. Giardina, "A robust digital baseband predistorter constructed using memory polynomials," Communications, IEEE Transactions on, vol. 52, pp. 159-165, 2004. 
[10] B. Widrow, J. M. McCool, M. G. Larimore, and C. R. Johnson, Jr.,

"Stationary and nonstationary learning characteristics of the LMS

adaptive filter," Proceedings of the IEEE, vol. 64, pp. 1151-1162, 1976.

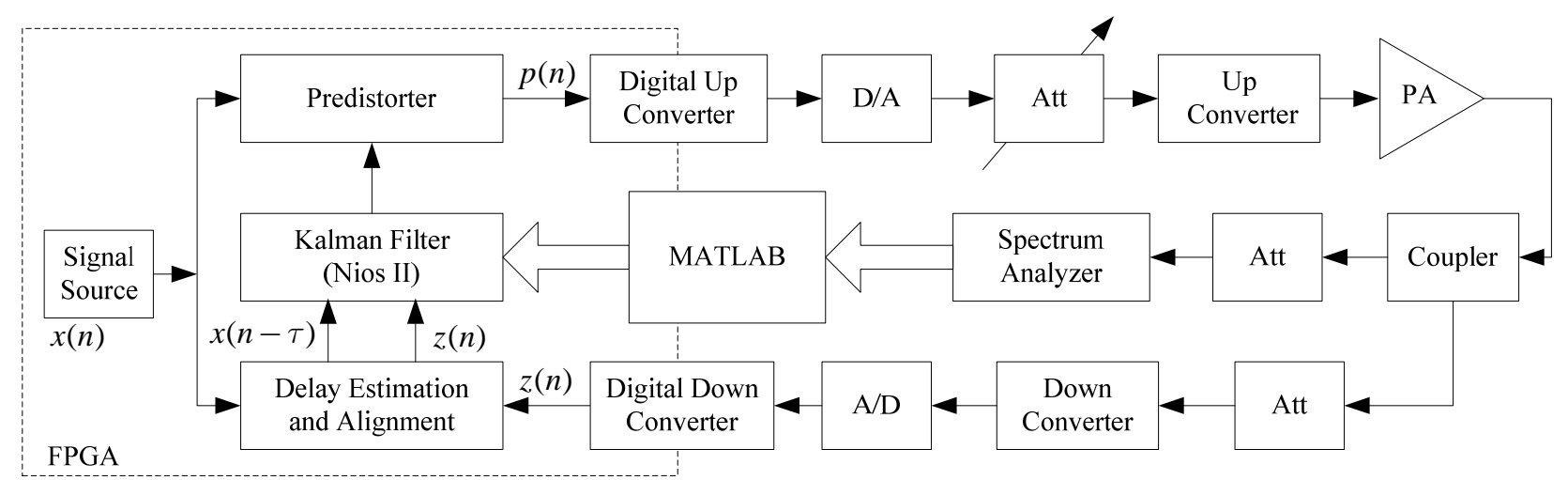

Figure.6 Hardware experiment setup. 